\title{
Effects of a mindfulness-based psychoeducation programme for Chinese patients with schizophrenia: 2-year follow-up
}

\author{
Wai Tong Chien and David R. Thompson
}

\section{Background}

Psychoeducation programmes for people with schizophrenia are shown to reduce relapses but few studies have indicated significant improvements in patients' illness awareness and insight, functioning, symptom severity or rates of readmission to hospital.

\section{Aims \\ To examine the effects of a mindfulness-based psychoeducation programme for chinese people with schizophrenia.}

\section{Method}

A multisite randomised controlled trial was conducted with 107 out-patients with schizophrenia: 36 and 35 received a 6-month mindfulness-based psychoeducation and a conventional psychoeducation programme, respectively, and 35 received routine care alone. Patient outcome measures were psychiatric symptom severity, psychosocial functioning, social support, insight into illness/treatment, and frequency and duration of readmissions to hospital (ClinicalTrials.gov: trial registration NCT01667601).

\section{Results}

The mindfulness-based psychoeducation group reported significantly greater improvements in psychiatric symptoms, psychosocial functioning, insight into illness/treatment and duration of readmissions to hospital over 24 months when compared with the other two groups.

\section{Conclusions}

Mindfulness-based psychoeducation appears to be a promising approach to treatment for Chinese patients with schizophrenia.

\section{Declaration of interest}

None.
Many people with schizophrenia continue to experience disabling residual and remitted symptoms, impaired functioning and high relapse rates in community care. Practice guidelines ${ }^{1,2}$ and systematic reviews ${ }^{3,4}$ recommend that psychoeducation in either an individual or group format, on a fortnightly to monthly basis, should be offered to patients with schizophrenia as it is consistently effective in reducing relapse and encouraging adherence to medication. However, as with other models of psychosocial intervention, the effects of psychoeducation on patients' awareness of and insight into their schizophrenia and other functional outcomes over the longer term are inconclusive. ${ }^{4}$ This may be because these interventions focused less attention on awareness of and insight into mental illness or its symptoms, and offer limited strategies for motivating and self-empowering patients in their illness management. ${ }^{3-5}$ Other methodological limitations of studies of psychosocial interventions include few multisite trials with diverse health outcomes, small sample sizes and high attrition rates. ${ }^{6}$ Mindfulness-based stress reduction programmes aim, through meditation and discussion, to change patients' relationships with thoughts and negative feelings - they can experience how mindful observation and acceptance of their illness is empowering and calming. ${ }^{5,7}$ These fortnightly ten-session programmes have been shown to empower patients' personal care, symptom management and control over distressing thoughts in severe depression and anxiety. ${ }^{8}$ Although a widespread perception, emanating from case studies, exists that mindfulness practices can elicit harmful reactions in individuals with acute psychosis, such as intense suffering from increased awareness of their illness condition, ${ }^{9}$ two recent feasibility trials have indicated its benefits for patients with psychosis. ${ }^{5,7}$ Our study aimed to evaluate the effect of mindfulness-based psychoeducation on mental state, rate of readmission to hospital, insight into illness, social support and functioning over a 24-month follow-up of Chinese patients with schizophrenia when compared with those receiving either conventional psychoeducation or usual care.

\section{Method}

The study was a multicentre randomised controlled trial with a repeated-measures design to compare the intervention outcomes in three groups of patients with schizophrenia (ClinicalTrials.gov: trial registration NCT01667601). The study was undertaken in Hong Kong between August 2010 and March 2013. Analysis of data was on an intention-to-treat basis and all participants were followed up over 24 months, irrespective of whether the intervention was completed or not.

\section{Participants and study settings}

Of approximately 1085 eligible patients with schizophrenia (15\% of this patient population) attending three out-patient clinics in the largest geographical region (New Territories) of Hong Kong, $515(48 \%)$ were successfully contacted. Of these, 450 (87\%) agreed to participate and $107(24 \%)$ were then randomly selected. Chinese out-patients with schizophrenia from the three clinics were eligible to participate if they: (a) had a diagnosis of schizophrenia according to DSM-IV criteria; ${ }^{10}$ (b) had a history of $\leqslant 5$ years of illness at recruitment; (c) were aged 18 years or over; and (d) were able to understand Chinese/Mandarin. Patients were excluded if there was comorbidity with another mental illness such as affective and organic brain disorders. From each clinic, those eligible patients who agreed to participate were listed in alphabetical order and then selected randomly from the list ( $n=35-36$ per clinic), using a computer-generated random numbers table.

Sample size was estimated on the basis of previous clinical trials of psychoeducation for Chinese patients with schizophrenia, 
in which symptom severity and relapse rate were the main outcomes. ${ }^{7,11,12}$ Sample size calculation indicated that 108 participants ( $n=36$ per group) were required to detect any statistically significant differences in psychiatric symptom severity and rates of readmission to hospital between three groups with effect sizes of 0.50 and 0.48 respectively, an alpha of 0.05 and a power of 0.80 , allowing for $20 \%$ potential attrition. ${ }^{13}$ With informed written consent and baseline measurement taken at recruitment, 107 patients were randomly assigned to either mindfulness-based psychoeducation $(n=36)$, conventional psychoeducation $(n=36)$ or usual care $(n=35,1$ withdrew before the intervention). Another 65 patients were approached but refused because of a lack of interest in participating $(n=28)$, an unwillingness to be known as having a mental illness $(n=20)$ and/or an inconvenient time for group meetings $(n=17)$.

\section{Procedure}

This trial was approved by the Human Subject Research Ethics Committee of the university and the study clinics and a flow diagram summarises the study procedures according to the revised version of the CONSORT statement (Fig. 1). ${ }^{14}$ After giving their written consent, patients were asked to draw a sealed opaque envelope, in which a labelled number card indicated the group to which they were assigned. An independent trained research nurse performed the outcome measurements using a set of questionnaires before treatment allocation (Time 1), at 1 week (Time 2), 12 months (Time 3) and 24 months (Time 4) following the 6-month interventions. Both the assessor and clinic staff were masked to treatment allocation.

\section{Measures}

At Times 1-4, the research nurse asked the participants to complete four questionnaires: the Brief Psychiatric Rating Scale (BPRS), ${ }^{15}$ Specific Levels of Functioning Scale (SLOF), ${ }^{16}$ 6-item Social Support Questionnaire $\left(\right.$ SSQ6) ${ }^{17}$ and Insight and Treatment Attitudes Questionnaire (ITAQ). ${ }^{18}$ Frequency and duration of readmissions to psychiatric hospital over the previous 6 or 12 months at Times 1-4 were collected from clinic records.

The 18-item BPRS has been used globally in clinical assessment and research to assess the severity of psychiatric symptoms. ${ }^{15}$ Items were rated on a seven-point Likert scale $(0$, not assessed; 1, not present; 6, extremely severe). The BPRS indicated satisfactory content validity, interrater reliability (intraclass correlation $(\mathrm{ICC})=0.89)$, and internal consistency (Cronbach's $\alpha=0.85$ ) in Chinese patients with psychosis. ${ }^{19}$

The 43-item SLOF measures the levels of patients' psychosocial functioning and comprises three functional domains for patients with schizophrenia: self-maintenance (12 items); social functioning (14 items); and community living skills (17 items). ${ }^{16}$ The Chinese version used in this study showed satisfactory content validity, test-retest reliability (Pearson's $r=0.79$ ), and internal consistency (Cronbach's $\alpha=0.90-0.96$ for subscales) in Chinese patients with schizophrenia. ${ }^{12,19}$

The SSQ6 was used to measure each patient's satisfaction with the social support available in his/her immediate social environment. ${ }^{17}$ The items are rated on a six-point Likert scale, with a higher total score (range 0-6) indicating more satisfaction with the available social support. The translated Chinese version indicated satisfactory content validity and internal consistency (Cronbach's $\alpha=0.89-0.93$ ) in Chinese patients with psychosis. ${ }^{11,19}$

The 11-item ITAQ ${ }^{18}$ measures patients' awareness of and insight into illness and need for treatment in schizophrenia. Its items are rated on a three-point Likert scale ( 0 , no insight; 1 , partial insight; and 2, good insight). The higher the score, the better is the patient's insight regarding recognition of illness-related problems and willingness to receive treatment. The Chinese version indicated satisfactory internal consistency (Cronbach's $\alpha=0.82)$, interrater reliability $(\mathrm{ICC}=0.82)$ and correlation (Pearson's $r=0.56$ and $0.60, P=0.001$ ) for symptom severity and psychopathology. ${ }^{20}$

Patients' demographic and clinical data (for example age, gender, education and duration of illness) were also collected at baseline. Dosages of antipsychotic medication examined from patients' out-patient progress sheets were converted into haloperidol equivalents for comparison. ${ }^{21}$

\section{Mindfulness-based psychoeducation programme}

Participants $(n=36)$ received 12 fortnightly, $2 \mathrm{~h}$ sessions of the mindfulness-based psychoeducation programme (MBPP group) (i.e. over 24 weeks), with 11-13 patients per group, in addition to usual psychiatric out-patient care (as described below). The programme was developed in accordance with recent psychoeducation programmes. ${ }^{11,22}$ as well as a mindfulness-based stress reduction programme tested in Chinese patients with depression. ${ }^{8}$ One nurse therapist led and facilitated patients to become more aware of and relate differently to their thoughts, feelings and sensations such as hallucinations and delusions, rather than identifying with them as accurate readouts on reality. The programme consisted of seven components in three phases (see online supplement):

(a) phase 1: orientation and engagement, empowerment and focused awareness of experiences, bodily sensations/thoughts and guided awareness exercises and homework practices;

(b) phase 2: education about schizophrenia care, intentionally exploring and dealing with difficulties regarding symptoms and problem-solving practices; and

(c) phase 3: behavioural rehearsals of relapse prevention strategies, accessible community support resources and future plans.

Individual participants were requested to regularly (daily) practice intentional and focused awareness of body sensations, thoughts and feelings and mindful walking in the early phases, and in later stages, select self-empowering and constructive perspectives for working with negative/distressing thoughts and feelings.

As mindfulness training served to increase patients' acceptance and management of their individual thoughts and emotional responses within their cultural context, the programme adopted a few strategies to address traditional Chinese cultural tenets. For instance, the first phase focused on understanding strong interdependence and inviting more mutual support and problem-solving among group members. In the second/third phases, patients were encouraged to cultivate an open, accepting mode of their responses to problems/stimuli and develop a 'decentred' attitude (recognising that the established beliefs and practices constitute only one of several possible interpretations) on their thoughts/feelings. ${ }^{8}$ Resolution around strong selfcenteredness (for example, saving face and reconstruction of self-image) was reinforced.

\section{Conventional psychoeducation programme}

In addition to usual out-patient care, participants in the conventional psychoeducation programme group (CPEP group, $n=36$ ) received a 6-month programme of conventional psychological support and education conducted by a trained psychiatric nurse experienced in leading psychiatric rehabilitation and education groups. Similar to the mindfulness-based psychoeducation 


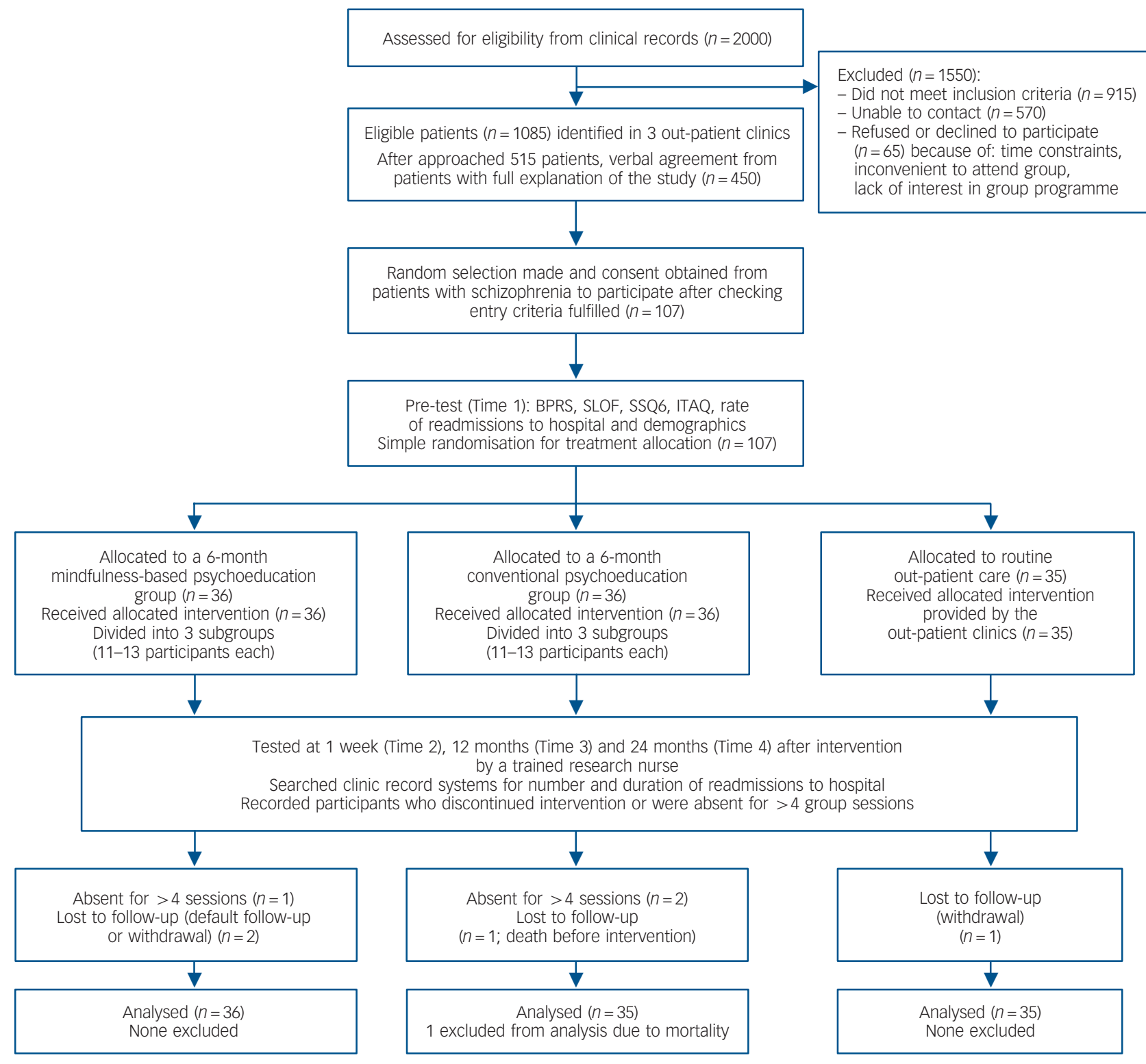

Fig. 1 Flow diagram of clinical trial for psychoeducation and usual care groups.

BPRS, Brief Psychiatric Rating Scale; SLOF, Specific Levels of Functioning Scale; SSQ6, 6-item Social Support Questionnaire; ITAQ, Insight and Treatment Attitudes Questionnaire.

programme, this group programme consisted of 12 fortnightly, $2 \mathrm{~h}$ sessions (11-13 participants per group). Its content was modified from other similar programmes 20,23 and comprised four stages: engaging and empowering individual patients (two sessions on orientation and engagement of participants and discussion about goals); education and survival skills workshop (four sessions covering basic facts on schizophrenia and stressful life situations and coping strategies); preventing relapse through problem-solving training (four sessions); and evaluation of knowledge and skills learnt, and preparation for the future (two sessions).

The psychiatric nurses in both the psychoeducation programmes were trained by the research team and a psychotherapist with a 3-day workshop and supervised practice within all group sessions. Progress monitoring of the two programmes was conducted between group sessions and consisted of regular reviews of audiotapes of each session by the research team, together with the nurse therapists, and clarification of any problems encountered.

\section{Usual out-patient care}

Participants in the usual care group $(n=35)$ received usual psychiatric out-patient services, which were similar across the three clinics. These services included monthly medical consultation and treatment by psychiatrists, advice and brief education on medication and community care services by psychiatric nurses, social welfare and financial assistance by medical social workers, and individual and family counselling by clinical psychologists whenever necessary.

\section{Data analyses}

All quantitative data collected were analysed on an intention-totreat basis using SPSS version 19.0 for Windows. Differences in demographic characteristics and the outcome measures (BPRS, SLOF, SSQ6, ITAQ and readmission to hospital rates) at Time 1 between the three study groups and between the three clinics under study within each of the three groups were assessed by 
analysis of variance (ANOVA) or the Kruskal-Wallis test ( $H$ statistic) as appropriate. Without any violation of assumptions of normality, linearity and homogeneity of variance-covariance and multicollinearity, ${ }^{13}$ multivariate analysis of variance (MANOVA) was performed for the dependent (outcome) variables to determine the treatment effects over time (group $\times$ time interactive effects), followed by univariate analyses (repeated-measures ANOVA) of the variables if the MANOVA test results were significant. Bonferroni's multistage procedure was used to adjust the significance level for the multiple ANOVA analyses (adjusted $P=0.01){ }^{24}$ The Helmert contrast codes were set to test any significant differences between groups on those measures that indicated a significant interactive effect of time $\times$ group in the repeated-measures ANOVA tests. ${ }^{13}$ Except for the repeatedmeasures ANOVA test, the level of statistical significance was set at 0.05 .

\section{Results}

\section{Sample characteristics}

Demographic and clinical characteristics of the three study groups and non-participants were similar (Table 1, see online Table DS1 for a more detailed version of this table), with no statistically significant differences $(P>0.15)$. More than half of the patients (56-60\%) were taking medium dosages of oral or intramuscular antipsychotic medications (haloperidol equivalent mean values ${ }^{21}$ were between $8.5 \mathrm{mg} /$ day $($ s.d. $=5.1)$ and $11.3 \mathrm{mg} /$ day $($ s.d. $=6.3)$ ). The mean duration of the illness was 2.6 years (range 3-60 months).

Three participants $(8.3 \%)$ in each of the MBPP and CPEP groups and one $(2.9 \%)$ in the usual care group either dropped out or were absent for more than 4 of the 12 group sessions. Reasons were similar, including lack of time/interest to attend, unstable mental state, and/or limited family encouragement and support. One CPEP participant died before the intervention and their data were excluded from the outcome analysis.

\section{Homogeneity of study groups}

There were no statistically significant differences in the amount and types of atypical $v$. conventional antipsychotics at Times 1-4 (ANOVA or $\chi^{2}$ tests, $P>0.10$ ) between the three groups (Table 1) nor in the baseline mean scores of the outcome measures $(F(5,104)=1.35, P>0.20)$ and between the three clinics within each of the study groups ( $H$ statistic: 1.2-1.8, $P>0.1$ ), or significant correlations $(r<0.20)$ between demographic characteristics and all of the outcome measures (BPRS, SLOF, SSQ6, ITAQ and frequency and duration of readmissions to hospital), indicating no covariate effects.

\section{Effects of interventions}

The multivariate analysis of the dependent variables (group $\times$ time) indicated a statistically significant difference between the three groups, $F(5,104)=5.98, P=0.001$ (Wilks' $\lambda=0.92$, partial $\eta^{2}=0.28$ ). With this significant multivariate test result, repeated-measures ANOVAs of each of the outcome variables were performed. Results (Table 2 and Fig. 2) indicated that there were statistically significant differences between the three groups: a reduction in the BPRS score $(F(2,104)=4.36, P<0.005)$ and duration of readmissions to hospital $(F(2,104)=4.80, \quad P<0.004)$, and an increase in the SLOF $(F(2,104)=4.98, P<0.004)$ and ITAQ scores $(F(2,104)=6.52$,

\begin{tabular}{|c|c|c|c|c|c|c|}
\hline \multirow[b]{2}{*}{ Characteristics } & \multicolumn{4}{|c|}{ Group } & \multirow[b]{2}{*}{$\begin{array}{c}\text { Test } \\
\text { value }^{\mathrm{b}}\end{array}$} & \multirow[b]{2}{*}{$P$} \\
\hline & $\begin{array}{l}\text { MBPP } \\
(n=36)\end{array}$ & $\begin{array}{c}\text { CPEP } \\
(n=36)\end{array}$ & $\begin{array}{l}\text { Usual care } \\
\qquad(n=35)\end{array}$ & $\begin{array}{l}\text { Non-participants } \\
\qquad(n=343)\end{array}$ & & \\
\hline \multicolumn{5}{|l|}{ Gender, $n(\%)$} & \multirow[t]{3}{*}{1.21} & \multirow[t]{3}{*}{0.34} \\
\hline Male & $20(55.6)$ & $21(58.3)$ & $20(57.1)$ & $198(57.7)$ & & \\
\hline Female & $16(44.4)$ & $15(41.7)$ & $15(42.9)$ & $145(42.3)$ & & \\
\hline Age, mean (s.d.) & $25.1(6.8)$ & $25.8(7.9)$ & $26.0(8.5)$ & $25.9(12.8)$ & 1.58 & 0.16 \\
\hline \multicolumn{5}{|l|}{ Education level, $n$ (\%) } & \multirow[t]{4}{*}{1.38} & \multirow[t]{4}{*}{0.19} \\
\hline Primary school or below & $8(22.2)$ & $8(22.2)$ & $8(22.9)$ & $75(21.9)$ & & \\
\hline Secondary school & $20(55.6)$ & $19(52.8)$ & $19(54.3)$ & $185(53.9)$ & & \\
\hline University or above & $8(22.2)$ & $9(25.0)$ & $8(22.9)$ & $83(24.2)$ & & \\
\hline Monthly household income, HK\$c: mean (s.d.) & $13240(1714)$ & $13010(1805)$ & $12805(1980)$ & $13345(2965)$ & 1.45 & 0.15 \\
\hline Duration of illness, years: mean (s.d.) & $2.6(1.7)$ & $2.5(1.8)$ & $2.7(1.8)$ & $2.5(2.1)$ & 1.32 & 0.26 \\
\hline \multicolumn{5}{|l|}{ Number of family members living with patient, $n(\%)$} & \multirow[t]{4}{*}{1.10} & \multirow[t]{4}{*}{0.38} \\
\hline $0-1$ & $13(36.1)$ & $14(38.9)$ & $13(37.1)$ & $130(37.9)$ & & \\
\hline $2-3$ & $18(50.0)$ & $18(50.0)$ & $17(48.6)$ & $165(48.1)$ & & \\
\hline $4-5$ & $5(13.9)$ & $4(11.1)$ & $5(14.3)$ & $48(14.0)$ & & \\
\hline \multicolumn{4}{|l|}{ Type of medication, $n$ (\%) } & & \multirow[t]{5}{*}{1.32} & \multirow[t]{5}{*}{0.24} \\
\hline Conventional antipsychotics (e.g. haloperidol) & $9(25.0)$ & $8(22.2)$ & $8(22.9)$ & $76(22.2)$ & & \\
\hline Atypical antipsychotics (e.g. risperidone) & $17(47.2)$ & $18(50.0)$ & $16(45.7)$ & $170(49.6)$ & & \\
\hline Antidepressants (e.g. fluoxetine) & 5 (13.8) & $4(11.1)$ & $4(11.4)$ & $40(11.7)$ & & \\
\hline Blended mode ${ }^{d}$ & $5(13.8)$ & $6(16.7)$ & $7(20.0)$ & $57(16.6)$ & & \\
\hline \multicolumn{5}{|l|}{ Dosage of medication, ${ }^{\mathrm{e}} \mathrm{n}(\%)$} & \multirow[t]{4}{*}{1.18} & \multirow[t]{4}{*}{0.32} \\
\hline High & $9(25.0)$ & $10(27.8)$ & 9 (25.7) & $83(24.2)$ & & \\
\hline Medium & $20(55.6)$ & $20(55.6)$ & $21(60.0)$ & $198(57.7)$ & & \\
\hline Low & 7 (19.4) & $6(16.7)$ & 5 (14.3) & $62(18.1)$ & & \\
\hline \multicolumn{7}{|c|}{$\begin{array}{l}\text { a. See online Table DS1 for a more detailed version of this table. } \\
\text { b. Analysis of variance }(F-\text {-test, d.f. }=448 \text { ) or the Kruskal-Wallis test by ranks }(H \text { statistic, d.f. }=3 \text { ) was used to compare the sociodemographic variables of patients among the three } \\
\text { study groups and the non-participants. } \\
\text { c. US\$1 = HK\$7.8. } \\
\text { d. Patients were taking more than one type of psychotropic medication such as the use of either conventional and atypical antipsychotic or atypical antipsychotic together with one } \\
\text { antidepressant. } \\
\text { e. Dosage levels of antipsychotic medication were compared with the average dosage of medication taken by schizophrenic patients in haloperidol-equivalent mean values. }{ }^{19}\end{array}$} \\
\hline
\end{tabular}




\begin{tabular}{|c|c|c|c|c|c|c|}
\hline \multirow[b]{2}{*}{ Measure (range) ${ }^{a}$} & \multicolumn{3}{|c|}{ Group, mean (s.d.) } & \multirow[b]{2}{*}{$F(2,104)$} & \multirow[b]{2}{*}{$P$} & \multirow{2}{*}{$\begin{array}{l}\text { Effect size } \\
\text { (partial } \eta \text { ) }\end{array}$} \\
\hline & $\operatorname{MBPP}(n=36)$ & CPEP $(n=35)^{\mathrm{b}}$ & Usual care $(n=35)$ & & & \\
\hline \multicolumn{4}{|l|}{ Insight and Treatment Attitudes Questionnaire (0-33) } & 6.52 & 0.001 & 0.28 \\
\hline Time 1 & $14.3(2.5)$ & $14.0(2.6)$ & $14.5(2.0)$ & & & \\
\hline Time 2 & $16.5(2.7)$ & $15.8(2.6)$ & $14.8(2.9)$ & & & \\
\hline Time 3 & $19.9(3.1)$ & $16.9(3.1)$ & $15.0(3.5)$ & & & \\
\hline Time 4 & $21.3(3.3)$ & $16.3(3.3)$ & $14.8(3.8)$ & & & \\
\hline \multicolumn{4}{|l|}{ Specific Level of Functioning (43-215) } & 4.98 & 0.004 & 0.19 \\
\hline Time 1 & $138.1(13.8)$ & $135.9(18.3)$ & $133.8(16.1)$ & & & \\
\hline Time 2 & $155.0(20.1)$ & $143.0(20.5)$ & $128.8(21.1)$ & & & \\
\hline Time 3 & $168.2(20.0)$ & $145.5(21.4)$ & $120.9(22.3)$ & & & \\
\hline Time 4 & $176.9(22.1)$ & $146.1(20.4)$ & $119.1(22.8)$ & & & \\
\hline \multicolumn{4}{|l|}{ Brief Psychiatric Rating Scale (0-108) } & 4.36 & 0.005 & 0.16 \\
\hline Time 1 & $30.6(7.5)$ & $32.0(6.7)$ & $31.6(5.2)$ & & & \\
\hline Time 2 & $24.8(6.0)$ & $28.9(5.1)$ & $30.9(6.8)$ & & & \\
\hline Time 3 & $20.1(5.9)$ & $26.8(8.1)$ & $34.0(6.9)$ & & & \\
\hline Time 4 & $17.0(4.9)$ & $28.1(7.0)$ & $35.8(7.8)$ & & & \\
\hline \multicolumn{4}{|l|}{ Six-item Social Support Questionnaire (0-30) } & 1.98 & 0.132 & 0.05 \\
\hline Time 1 & $4.0(1.1)$ & $4.1(1.1)$ & $4.0(0.9)$ & & & \\
\hline Time 2 & $4.1(1.3)$ & $4.2(1.5)$ & $4.0(1.3)$ & & & \\
\hline Time 3 & $3.8(1.6)$ & $3.7(1.1)$ & $3.6(1.8)$ & & & \\
\hline Time 4 & $3.6(1.6)$ & $3.9(1.0)$ & $3.9(1.5)$ & & & \\
\hline \multicolumn{4}{|l|}{ Number of readmissions to hospital ${ }^{c}$} & 2.78 & 0.096 & 0.09 \\
\hline Time 1 & $2.8(1.6)$ & $2.5(0.9)$ & $2.7(1.0)$ & & & \\
\hline Time 2 & $2.4(1.3)$ & $2.3(1.1)$ & $2.6(1.2)$ & & & \\
\hline Time 3 & $2.1(0.9)$ & $2.5(1.0)$ & $2.9(1.2)$ & & & \\
\hline Time 4 & $1.8(1.3)$ & $2.3(1.1)$ & $3.0(1.3)$ & & & \\
\hline \multicolumn{4}{|l|}{ Duration of readmissions to hospital ${ }^{d}$} & 4.80 & 0.004 & 0.16 \\
\hline Time 1 & $19.0(4.3)$ & $18.5(4.7)$ & $19.2(4.1)$ & & & \\
\hline Time 2 & $15.7(4.1)$ & $16.2(4.1)$ & $19.8(4.8)$ & & & \\
\hline Time 3 & $13.0(3.6)$ & $16.5(5.0)$ & $20.2(5.2)$ & & & \\
\hline Time 4 & $11.0(4.0)$ & $16.1(6.0)$ & $21.0(6.9)$ & & & \\
\hline \multicolumn{7}{|c|}{$\begin{array}{l}\text { MBPP, mindfulness-based psychoeducation programme; CPEP, conventional psychoeducation programme; Time 1, baseline measurement at the start of intervention; Time } 2,1 \text { week } \\
\text { after intervention; Time } 3,12 \text { months after intervention; Time } 4,24 \text { months after intervention. } \\
\text { Results in bold are significant. } \\
\text { a. POssible range of scores of each scale. } \\
\text { b. One of the } 36 \text { participants in the CPEP group died before the intervention and was therefore excluded from data analysis. } \\
\text { c. Average number of readmissions to a psychiatric in-patient unit over } 6 \text { or } 12 \text { months at Times } 1 \text { to } 4 .\end{array}$} \\
\hline
\end{tabular}

$P<0.001)$ using a Bonferroni adjusted alpha level of 0.01. An examination of the adjusted mean scores at Times 1-4 (Fig. 2) indicated that the MBPP group reported more consistently positive improvements in ITAQ, SLOF and BPRS scores and duration of readmissions to hospital than the CPEP group. The usual care group reported mainly progressive mild negative changes of scores in all outcomes over the 2-year follow-up and a significant reduction in the SLOF and BPRS scores at Time 3.

There were also significant statistical differences on the mean scores of the three SLOF subscales between the three groups at Times 2-4. (Fig. 3). The MBPP group reported significantly greater improvements in self-maintenance $(F(2,104)=4.83)$, social functioning, $\quad(F(2,104)=4.28)$ and community living skills $(F(2,104)=4.32)$ and all of their $P$-values were $<0.005$ and with effect sizes $\left(\eta^{2}\right)$ of 0.17 to 0.20 (large effects). ${ }^{24}$

Helmert's contrasts indicated that the mean differences between groups at two time-points on the following outcomes were significant at 0.05 , and contributed to the overall multivariate significance.

(a) The ITAQ score of the MBPP group increased more significantly from Times 1 to 4 than in the other two groups (MBPP v. CPEP group, mean differences were 0.7, 3.0 and 5.0 (s.e. $=0.3-1.9$ ) and MBPP $v$. usual care group, mean differences were $1.7,4.9$ and 6.5 (s.e. $=0.6-2.4)$ at Times $2-4$, respectively), whereas for the CPEP group it increased slightly over time. (b) The SLOF score of the MBPP group increased more significantly from Times 1 to 4 (MBPP $v$. CPEP group, mean differences were 12.0, 22.7 and 30.8 (s.e. $=3.0-4.9$ ) and MBPP $v$. usual care group, mean differences were 26.2, 47.3 and 57.8 (s.e. $=4.8-7.6$ ) at Times $2-4$, respectively). For the CPEP group it also increased and differed significantly from the usual care group, which showed a consistent reduction over time.

(c) The BPRS score of the MBPP group increased more significantly from Times 1 to 4 (MBPP $v$. CPEP group, mean differences were 4.1, 6.7 and 11.1 (s.e. $=0.9-3.0$ ) and MBPP $v$. usual care group, mean differences were 6.1, 13.9 and 18.8 (s.e. $=1.9-4.5)$ at Times $2-4$, respectively). For the CPEP group it also increased and differed significantly from the usual care group at Times 2 to 3 .

(d) The duration of readmissions to hospital in the MBPP group were significantly reduced from Times 1 to 4 (MBPP $v$. CPEP group, mean differences (days) were $0.5,3.5$ and 5.1 (s.e. $=0.2-1.8)$ and MBPP $v$. usual care group, mean differences were 4.1, 7.2 and $10.0($ s.e. $=1.2-4.9)$ at Times $2-4$, respectively). For the CPEP group it was slightly reduced and for the usual care group it consistently increased.

There were progressive mild but non-significant reductions in medication scores among the three groups based on the converted haloperidol equivalents, ${ }^{21}$ from a mean of $11.8-12.1 \mathrm{mg} /$ day (s.d.=3.8-4.2) at Time 1 , a mean of $10.8-11.9 \mathrm{mg} /$ day 
(a)

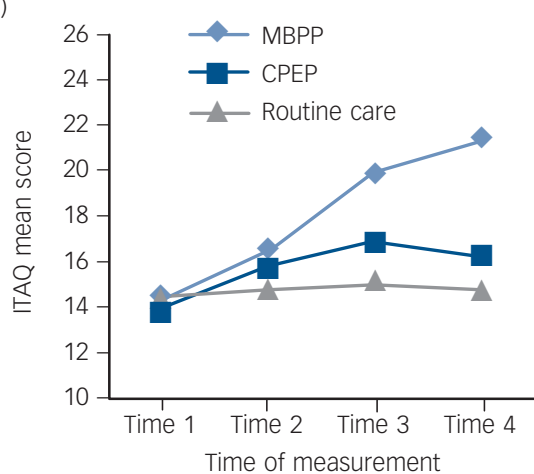

(d)

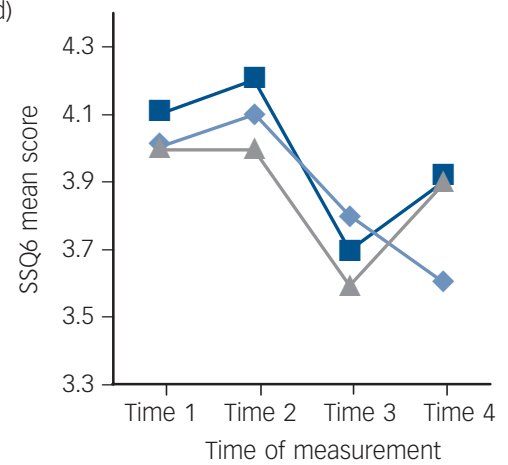

(b)

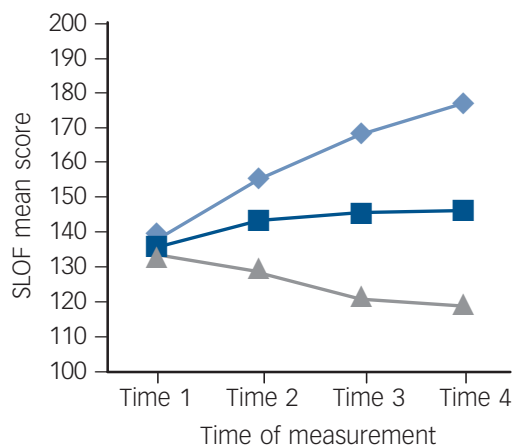

(e)

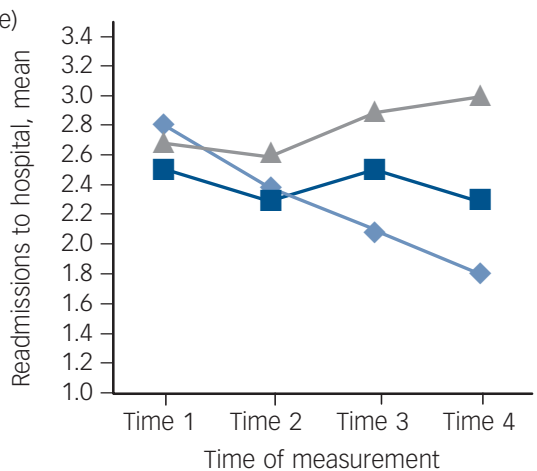

(C)

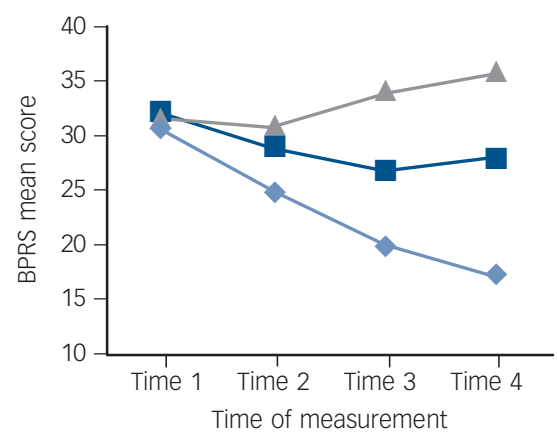

$(f)$

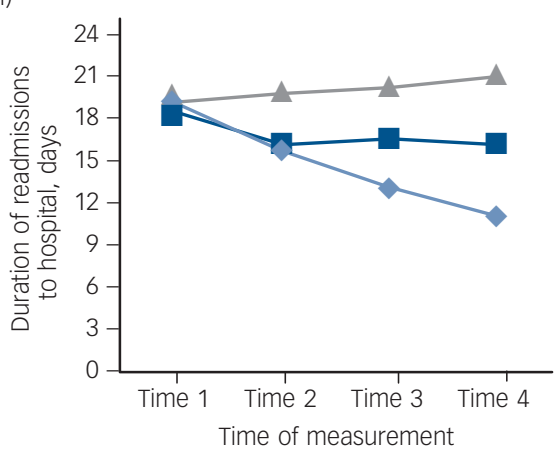

Fig. 2 Mean scores of study outcomes at baseline (Time 1) to 24-month follow-up (Time 4).

Mean scores on the (a) Insight and Treatment Attitudes Questionnaire (ITAQ); (b) Specific Levels of Functioning Scale (SLOF); (c) Brief Psychiatric Rating Scale (BPRS); (d) Six-item Social Support Questionnaire (SSQ6); (e) number of readmissions to hospital; ( $f$ ) duration of readmissions to hospitals (days). Mindfulness-based psychoeducation programme (MBPP) group $(n=36)$; conventional psychoeducation programme (CPEP) group $(n=35)$; routine care group $(n=35)$. Time 2, 1-week follow-up; Time 3, 12-month follow-up.

(s.d. $=4.1-4.8)$ at Time 2 , a mean of $10.6-11.7 \mathrm{mg} /$ day (s.d. $=4.0-$ 4.6) at Time 3, to a mean of $10.2-11.2 \mathrm{mg} /$ day (s.d. $=4.1-4.8$ ) at Time 4. There was also no significant difference in the types/ frequency of participation in other individual/family-based psychological treatments (two-way ANOVA, $P>0.1$ ).

\section{Discussion}

\section{Benefits of mindfulness-based psychoeducation}

The findings from this study strongly suggest that mindfulnessbased psychoeducation for schizophrenia appears to be a promising approach to the treatment of this condition. A 6-month programme of this intervention, when compared with conventional psychoeducation or usual care, resulted in better patient outcomes (severity of psychiatric symptoms, level of psychosocial functioning, awareness of and insight into illness and need for treatment and duration of readmissions to hospital) at 2-year follow-up. Interestingly, there were no statistically significant differences between the three groups in terms of satisfaction with social support or frequency of readmissions to hospital. With a paucity of clinical trials reported, these findings suggest that mindfulness-based psychoeducation can be effective not only in patients with recurrent depression, ${ }^{8}$ but also in people with schizophrenia and probably other psychotic disorders. ${ }^{5}$

This mindfulness-based psychoeducation programme is one of very few that has attempted to enhance awareness and acceptance among Chinese people with schizophrenia and that addresses their specific cultural needs (such as self-blame about having mental illness and interdependence among co-patients). ${ }^{7}$ Similar to mindfulness-based interventions in other clinical trials for depression, anxiety ${ }^{8,25}$ and psychotic disorders, ${ }^{5,9}$ this programme can enhance psychotic patients' positive thoughts and relieve their guilty feeling towards the illness and its symptoms. Recent literature on psychiatric treatments cautions against the use of mindfulness meditation training for people with psychosis because of concerns about the potential for exacerbating psychotic symptoms during meditation practice. ${ }^{6,7}$ Nevertheless, this clinical trial provides evidence attesting to this model of intervention improving these patients' awareness of and insight into their illness, treatment needs and clinical functioning, supporting the findings of a feasibility trial in the UK of mindfulness groups for patients with distressing voices and paranoia. $^{5}$ This clinical trial also demonstrates the significant longer-term (2-year) beneficial effects of a mindfulness-based programme. Whether these improvements are a result of a combined effect of mindfulness training and psychoeducation, all of which are considered potential therapeutic factors in different current models of psychosocial intervention, ${ }^{3,11}$ remains to be answered. Thus, further research to explore the therapeutic effects of the individual active elements in the programme is recommended. In addition, there is a suggestion that it may be particularly suitable for Chinese people who are self-conscious and internalising their emotions and feelings. ${ }^{8}$ The mindfulnessbased programmes adopted in this trial and a few recent studies are all highly structured and demanding for participants and include work on home assignments using practices such as selfawareness of their body, redirecting and increasing their focus on symptoms and behaviours. ${ }^{5,8,26}$ These extensive assignments may not be easily implemented by patients with florid psychotic symptoms and an unstable mental state, or with chronic illness and negative symptoms. Therefore, the flexibility of the approach used should be studied further. 

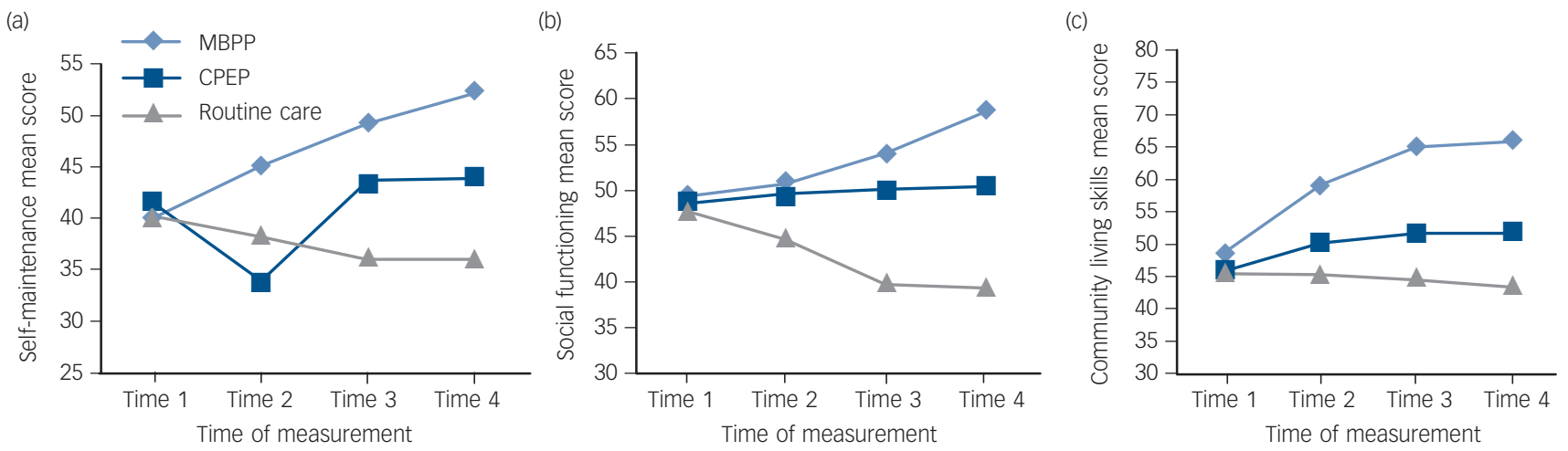

Fig. 3 Mean scores on three subscales of the Specific Level of Functioning (SLOF) scale at baseline (Time 1) to 24-month follow-up (Time 4).

(a) Self-maintenance; (b) social function; and (c) community living skills subscales. Mindfulness-based psychoeducation programme (MBPP) group ( $n=36)$; conventional psychoeducation programme (CPEP) group $(n=35)$; routine care group $(n=35)$. Time 2, 1-week follow-up; Time 3, 12-month follow-up.

\section{Why a mindfulness-based psychoeducation group?}

Mindfulness meditation training has been found to be effective in empowering patients to manage their distressing and negative thoughts and beliefs across a range of mental and physical health conditions, as well as demonstrating an encouraging prophylactic effect on illness relapse or recurrence in depressive and anxiety disorders. $^{8,25}$ However, there are few studies reporting the usefulness of mindfulness meditation (trained voluntary deployment attention) for people with schizophrenia or psychosis to increase patients' awareness of, and change their relationship with, unwanted thoughts, sensations and negative feelings. Our study reports similar positive outcomes to those found in all 15 participants with schizophrenia interviewed in a pilot study ${ }^{26}$ testing a mindfulness-based intervention to reduce anxiety: more awareness of and relief from their psychotic and other psychological symptoms, cognitive changes (such as more acceptance of and higher motivation in illness management) and a focus on the present. The findings from our trial suggest that people with schizophrenia may not be distressed by the occurrence of psychotic symptoms but by the meaning they construct and perceive, as suggested by recent qualitative studies. ${ }^{5,9,26}$ Further study on distress and the related behaviours associated with psychotic symptoms and the meditation role of cognition in driving or combating this distress is recommended. It is also noteworthy that the confounding effects of covariants such as medication adherence and other psychotherapeutic interventions were not examined in our study, thus providing directions for future research.

\section{Limitations of the study}

Only Chinese patients were recruited from three psychiatric outpatient clinics in one geographical region in Hong Kong. Although the participants in this study were randomly selected in these three clinics, most were volunteers who may have been highly motivated to participate in the group interventions, thus resulting in very low drop-out rates. These patients, whose schizophrenia was of short duration (mean 2.6 years), might not be representative of those with long-term or chronic schizophrenia, or of those with comorbidities of other mental disorders receiving mental healthcare services. Therefore, such selective sampling should be taken into consideration when comparisons are made between this and other studies of psychoeducation programmes. Unlike the samples in some studies on psychoeducation groups, it is also important to note that more than half of patients recruited in this study were male, taking low/medium dosages of antipsychotics, living with their family, and had a high level of education. In addition, the participants were not masked to the intervention undertaken and any Hawthorne effect among the participants could not be excluded. Also the programme was time-limited to 6 months and used a less standardised format than other psychoeducation programmes; and no booster session was offered.

\section{Implications for further research}

As the first pragmatic trial designed to evaluate the effect of a mindfulness-based psychoeducation group for young Chinese people with schizophrenia, these results lend support for further evaluation of this intervention as a treatment in other populations and settings. This may necessitate tailoring the structure and content of the programme to, for example, the cultural tenets of specific patient populations and evaluating its medium- to longterm effect. An exploration of not only individual participants' perceptions of and satisfaction with the intervention but also the group process, in terms of enablers and barriers and degree of involvement, ${ }^{16}$ is essential to better understand the active ingredients of a mindfulness-based psychoeducation group.

In this study, the mindfulness-based psychoeducation programme had substantial positive effects for patients with schizophrenia in terms of psychiatric symptom severity, psychosocial functioning, awareness of and insight into illness and need for treatment and duration of readmissions to hospital. However, there were no significant effects on patients' dosage of medication, service utilisation or perceived social support. Other longer-term benefits/outcomes used in psychoeducation studies such as improvement in global functioning, satisfaction with mental health services and quality of life warrant further investigation. ${ }^{4}$

Finally, in view of this intervention being shown to be effective, it will be interesting to observe whether further studies will replicate these findings and whether the intervention will be adopted more widely. There is a suggestion that it may be particularly suitable for Chinese people and we await with interest to see whether it is found to be so for other populations. Further research is needed to examine issues such as the flexibility of the approach used and whether the duration of the intervention has an impact on medication use. Therefore, it is important to investigate long-term effects, cost-effectiveness, and/or comparative effectiveness with other psychosocial interventions for schizophrenia, in larger representative samples from different socioeconomic and ethnic/ cultural backgrounds with different durations of illness and/or comorbidities of other mental disorders. 


\section{Clinical implications}

This mindfulness-based psychoeducation programme, when compared with a conventional psychoeducation programme and usual psychiatric out-patient care, appeared to be an effective alternative community-based intervention for Chinese people with schizophrenia. It was found to enhance patients' awareness of and insight into their illness and treatments and reduce the duration of their readmissions to hospitals and, similar to other psychoeducation groups, can improve patients' symptoms and functioning. The programme can yield improvements without any increased demand for community mental health services or medication use.

Wai Tong Chien, PhD, MPhil, BN, RMN, FPHKAN, School of Nursing, Faculty of Health and Social Sciences, The Hong Kong Polytechnic University, Hung Hom, Kowloon, Hong Kong, China; David R. Thompson, PhD, MBA, BSC, RN, FRCN, FAAN, FESC, Cardiovascular Research Centre, Australian Catholic University and Department of Psychiatry, University of Melbourne, Melbourne, Australia

Correspondence: Wai Tong Chien, Mental Health Research Group, School of Nursing, Faculty of Health and Social Sciences, The Hong Kong Polytechnic University, Hung Hom, Kowloon, Hong Kong SAR, China. Email: wai.tong.chien@polyu.edu.hk

First received 2 Jul 2013, final revision 11 Dec 2013, accepted 17 Feb 2014

\section{Funding}

This research was supported by the Central Research Fund, The Hong Kong Polytechnic University, and Health and Medical Research Fund, Hong Kong SAR, China.

\section{Acknowledgements}

Special thanks are given to the patients who participated in this study. The support and cooperation of mental health staff in the clinics under study is gratefully acknowledged.

\section{References}

1 Lehman AF, Lieberman JA, Dixon LB, McGlashan TH, Miller AL, Perkins DO, et al. Practice guideline for the treatment of patients with schizophrenia. Am J Psychiatry 2004; 161 (suppl 2): s1-56.

2 National Institute for Health and Clinical Excellence. Core Interventions in the Treatment and Management of Schizophrenia in Adults in Primary and Secondary Care (Update). NICE, 2009.

3 Rummel-Kluge C, Kissling W. Psychoeducation in schizophrenia: new developments and approaches in the field. Curr Opin Psychiatry 2008; 21: 168-72.

4 Xia J, Merinder LB, Belqamwar MR. Psychoeducation for schizophrenia. Cochrane Database Syst Rev 2001; 6: CD002831.

5 Chadwick P, Hughes S, Russell D, Russell I, Daqnan D. Mindfulness groups for distressing voices and paranoia: a replication and randomized feasibility trial. Behav Cogn Psychother 2009; 27: 403-12.
6 Bäuml J, Froböse T, Kraemer S, Rentrop M, Pitschel-Walz G. Psychoeducation: a basic psychotherapeutic intervention for patients with schizophrenia and their families. Schizophr Bull 2006; 32 (suppl 1): s1-9.

7 Chien WT, Lee YMI. The mindfulness-based psychoeducation program for Chinese patients with schizophrenia. Psychiatr Serv 2013; 64: 376-9.

8 Ma SH, Teasdale JD. Mindfulness-based cognitive therapy for depression: replication and exploration of differential relapse prevention effects. $J$ Couns Clin Psychol 2004; 72: 31-40.

9 Freeman D, Garety PA, Kuipers E, Fowler D, Bebbington PE. A cognitive model of persecutory delusions. Br J Clin Psychol 2002; 41: 331-47.

10 American Psychiatric Association. Diagnostic and Statistical Manual of Mental Disorders (4th edn). APA, 1994.

11 Chan SW, Yip B, Tso S, Cheng BS, Tam W. Evaluation of a psychoeducation program for Chinese patients with schizophrenia and their family caregivers. Patient Educ Couns 2009; 75: 67-76.

12 Li Z, Arthur D. Family education for people with schizophrenia in Beijing China: randomised controlled trial. Br J Psychiatry 2005; 187: 339-45.

13 Stevens JP. Applied Multivariate Statistics for the Social Sciences. Lawrence Erlbaum, 2002.

14 Schultz KF, Altman DG, Moher D, CONSORT Group. CONSORT 2010 statement: updated guidelines for reporting parallel group randomized trials. Ann Intern Med 2010; 152: 726-32.

15 Overall JE, Gorham DR. The Brief Psychiatric Rating Scale. Psychol Reports 1962; 10: 799-812.

16 Schneider LC, Struening EL. SLOF: a behavioral rating scale for assessing the mentally ill. Soc Work Res Abstr 1983; 19: 9-21.

17 Sarason IG, Sarason BR, Shearin EN, Pierce GR. A brief measure of social support: practical and theoretical implications. J Soc Person Relat 1987; 4 497-510.

18 McEvoy JP, Apperson L, Appelbaum PS, Ortlip P, Brecosky J, Hammill K, et al. Insight in schizophrenia: its relationship to acute psychopathology. J Nerv Ment Dis 1989: 177: 43-7.

19 Chien W-T, Chan SWC, Thompson DR. Effects of a mutual support group for families of Chinese people with schizophrenia: 18-month follow-up. Br J Psychiatry 2006; 189: 41-9.

20 Chien WT, Leung SF. A controlled trial of a needs-based, nurse-led psychoeducation program for Chinese patients with first-onset mental disorders: 6 month follow-up. Int J Nurs Practice 2013; 19 (suppl 1): s3-13.

21 Bezchlibnyk-Butler KZ, Jeffries JJ, Virani AS. Clinical Handbook of Psychotropic Drugs (17th edn). Hogrefe and Huber, 2007.

22 Kreyenbuhl J, Buchanan RW, Dickerson FB, Dixon LB, Schizophrenia Patients Outcomes Research Team. The Schizophrenia Patient Outcomes Research Team (PORT): updated treatment recommendations 2009. Schizophr Bull 2010; 36: 94-103.

23 Macpherson R, Jerrom B, Hughes A. A controlled study of education about drug treatment in schizophrenia. Br J Psychiatry 1996; 168: 709-17.

24 Tabachnick BG, Fidell LS. Using Multivariate Statistics. Allyn and Bacon, 2001.

25 Chlesa A, Serretti A. Mindfulness-based cognitive therapy for psychiatric disorders: a systematic review and meta-analysis. Psychiatry Res 2011; 187 441-53.

26 Brown LF, Davis LW, LaRocco VA, Strasburger A. Participant perspectives on mindfulness meditation training for anxiety in schizophrenia. Am J Psychiatric Rehabil 2010; 13: 224-42. 